\title{
PROBLEMAS DE ASSISTENNCIA MÉDICO-SANITÁRIA NO ESTADO DE SÃO PAULO, BRASIL
}

\author{
Ernesto Lima Gonçalves* \\ Evelyn Naked Castro Sá ** \\ Gastão E. de Arruda Camargo *** \\ João Yunes **** \\ Otávio Mercadante** \\ Ruy M. Gomes Pinto ***** \\ Sergio Reis Quaglia****** \\ Tristão Pereira da Fonseca F. ${ }^{* * *}$ \\ Zilah Wendel Abramo**
}

RSPU-B $/ 258$

Lima Gonçalves, E. et al. - Problemas de assistencia médıco-sanitária no Estado de São Paulo, Brasil. Rev. Saúde públ., S. Paulo, 9:181-90, 1975

RESUMO: Apresenta-se uma análise dos principais problemas médico-sumtârios do Estado de São Paulo, Brasil. Verificou-se a possibilidade de definição de 5 regiões, cujas caracteristicas são apreciavelmente homogêneas: a primeira, representada pelas sub-regiões de São José do Rio Preto, Araçatuba e Presidente Prudente; a segunda, correspondente a Bauru e Marilia; a terceira, inclui as áreas de Sorocaba, do Litoral e do Vale do Paraíba; a quarta, abrange Campinas e Ribeirão Preto; e a quinta, correspondente à Grande são Paulo. Para cada uma dessas regiões foram descritos os indicadores básicos de saude $e$ alguns aspectos relativos ao equipamento de saúde disponivel. Verificou-se a dificuldade de relacionar os dois tipos de indicadores. Foram examinados os problemas básicos relativos ao atendimento da população urbana $e$ da que habita as regióes rurais do Estado de São Paulo.

UNitermos: Assistência médico-sanitária. Niveis de saúde, indicadores. São Paulo, Brasil.

Quando se analisam os níveis de saúde da população do Estado de São Paulo. indicados pelos dados disponíveis para o conjunto da comunidade estadual, verifica-se que a situação está longe de ser sa- tisfatória, o que contrasta com a pujança e o nível de desenvolvimento da economia paulista. Daí caber aqui uma reflexão sobre os condicionamentos que impediram melhores resultados.

* Da Faculdade de Medicina da Universidade de São Paulo - Av. Dr. Arnaldo, 455 São Paulo, SP - Brasil

** Da Secretaria de Estado da Saúde - Av. São Luiz, 99 - São Paulo, SP — Brasil

*** Da Secretaria de Higiene e Saúde da Prefeitura do Município le São Paulo - Hospital Municipal - Rua Castro Alves, 60 - São Paulo, SP — Brasil

**** Da Faculdade de Saúde Pública da Universidade de São Paulo - Av. Dr. Arnaldo, 715 - São Paulo, SP - Brasil

*****: Da Faculdade de Arquitetura e Urbanismo da Universidade de São Paulo - Cidade Universitária "Armando Salles de Oliveira" - São Paulo, SP — Brasil

******* Do Instituto de Assistência Médica do Servidor Público Estadual - Rua Pedro de Toledo, 1800 - São Paulo, SP - Brasil 
LIMA GONÇALVES, E. et al. - Problemas de assistência médico-sanitária no Estado de São Paulo, Brasil. Rev. Saude publ., S. Paulo, 9:181-90, 1975.

TABELA 1

Distribuição da população por sexo, segundo a laixa etária, do Estado de São Paulo, 1970

\begin{tabular}{cr|r|r}
\hline Faixa etária & Homens & Mulheres & Total \\
\hline 0 a 4 anos & 1.111 .684 & 1.062 .752 & 2.177 .436 \\
5 a 9 anos & 1.146 .229 & 1.109 .277 & 2.255 .506 \\
10 a 14 anos & 1.046 .436 & 1.026 .215 & 2.072 .651 \\
15 a 19 anos & 921.658 & 941.702 & 1.863 .360 \\
20 a 49 anos & 3.631 .900 & 3.538 .796 & 7.170 .696 \\
50 a 69 anos & 894.510 & 896.247 & 1.790 .757 \\
70 e mais anos & 168.136 & 192.358 & 360.494 \\
Idade ignorada & 20.807 & 19.379 & 40.186 \\
Total & 8.931 .360 & 8.789 .726 & 17.721 .086
\end{tabular}

Fonte: Secretaria do Planejamento do Estado de São Paulo (SEPLAN), 1974

Uima primeira linha de condicionamentos é representada por dados de ordem demográfica; alguns fatos devem ser aqui salientados. Inicialmente, a composição etária da população paulista ao lado de sua distribuição pelos dois sexos (Tabela 1).

Verifica-se na Tabela 1 que $36,7 \%$ da população paulista situam-se na faixa etária entre 0 e 14 anos e que a população feminina acima dessa idade corresponde a $31,5 \%$ do total geral. A soma desses dois percentuais, atingindo $68,2 \%$ da população do Estado de São Paulo, justifica amplamente todos os programas de assistência à criança e à mulher que se possam desenvolver.

Outro dado sugestivo refere-se à intensa polarização que os grandes centros urbanos desenvolvem sobre a população em geral, o que faz com que o natural fenômeno de urbanização, inerente ao processo de desenvolvimento econômico e industrial, venha ocorrendo de maneira inadequada.
A conseqüência é a formação de grandes aglomerações populacionais, onde, ao lado de se desenvolverem recursos assistenciais de maior porte e mais alto grau de sofisticação, acumulam-se maiores problemas de saúde, sobretudo os ligados à infra-estrutura sanitária. Apenas a título exemplificativo podemos tomar dados relativos ao atendimento da população dos centros urbanos, no que se refere a redes de água encanada e de esgotos.

A Tabela 2 apresenta a relação entre tamanho das cidades e população abastecida por água encanada.

Verifica-se pelo exame da Tabela 2 que a percentagem de cidades onde já se atingiu a meta de $80 \%$ da população urbana abastecida por água encanada, reduz-se à medida que aumenta o porte da cidade.

Os dados relativos a esgotos figuram na Tabela 3 .

A análise da Tabela 3 demonstra que, também no que se refere à rede de esgoto, à medida que aumenta o porte da po- 
LIMA GONCALVES, E. et al. - Problemas de assistência médico-sanitária no Estado de São Paulo, Brasil. Rev. Saude puibl., S. Paulo, 9:181-90, 1975.

TABLA 2

Relação entre tamanho das cidades do Estado de São Paulo e população abastecida por água encanada, 1972

\begin{tabular}{|c|c|c|c|}
\hline $\begin{array}{c}\text { Tamanho das cidades } \\
\text { (n. }{ }^{\circ} \text { habitantes) }\end{array}$ & $\begin{array}{l}\text { Percentagem da população } \\
\text { abastecida }\end{array}$ & $\begin{array}{l}\text { Número de } \\
\text { cidades }\end{array}$ & $\begin{array}{l}\text { Percentagem } \\
\text { do n.o total }\end{array}$ \\
\hline \multirow{5}{*}{6.000} & 80.0 & 169 & 58,8 \\
\hline & 50,0 a 80,0 & 66 & 22,9 \\
\hline & menos de $\mathbf{5 0 , 0}$ & 20 & 6,9 \\
\hline & não tem & 33 & 11,4 \\
\hline & & 288 & 100,0 \\
\hline \multirow{5}{*}{6.001 a 12.000} & 80,0 & 46 & 59,7 \\
\hline & 50,0 a 80,0 & 30 & 38,9 \\
\hline & menos de 50,0 & - & - \\
\hline & não tem & 1 & 1,4 \\
\hline & & 77 & 100,0 \\
\hline \multirow{4}{*}{12.001 a 25.000} & 80,0 & 37 & 60,6 \\
\hline & 50,0 a 80,0 & 22 & 36,1 \\
\hline & menos de $\mathbf{5 0 , 0}$ & 2 & 3,3 \\
\hline & & 61 & 100,0 \\
\hline \multirow{4}{*}{25.001 a 50.000} & 80,0 & 17 & 48,6 \\
\hline & 50,0 a 80,0 & 18 & 51.4 \\
\hline & menos de 50,0 & - & - \\
\hline & & 35 & 100,0 \\
\hline \multirow{4}{*}{ mais de 50.000} & 80,0 & 12 & 50,0 \\
\hline & 50,0 a 80,0 & 10 & 41,7 \\
\hline & menos de 50,0 & 2 & 8,3 \\
\hline & & 24 & 100,0 \\
\hline
\end{tabular}

Fonte: Fundo Estadual de Saneamento Básico (FESB), 1974 
LIMA GONÇALVES. E. et al. - Problemas de assistência médico-sanitáiı no Estado de São Paulo, Brasil. Rev. Saúle pübl., S. Paulo, 9:181-90, 1975.

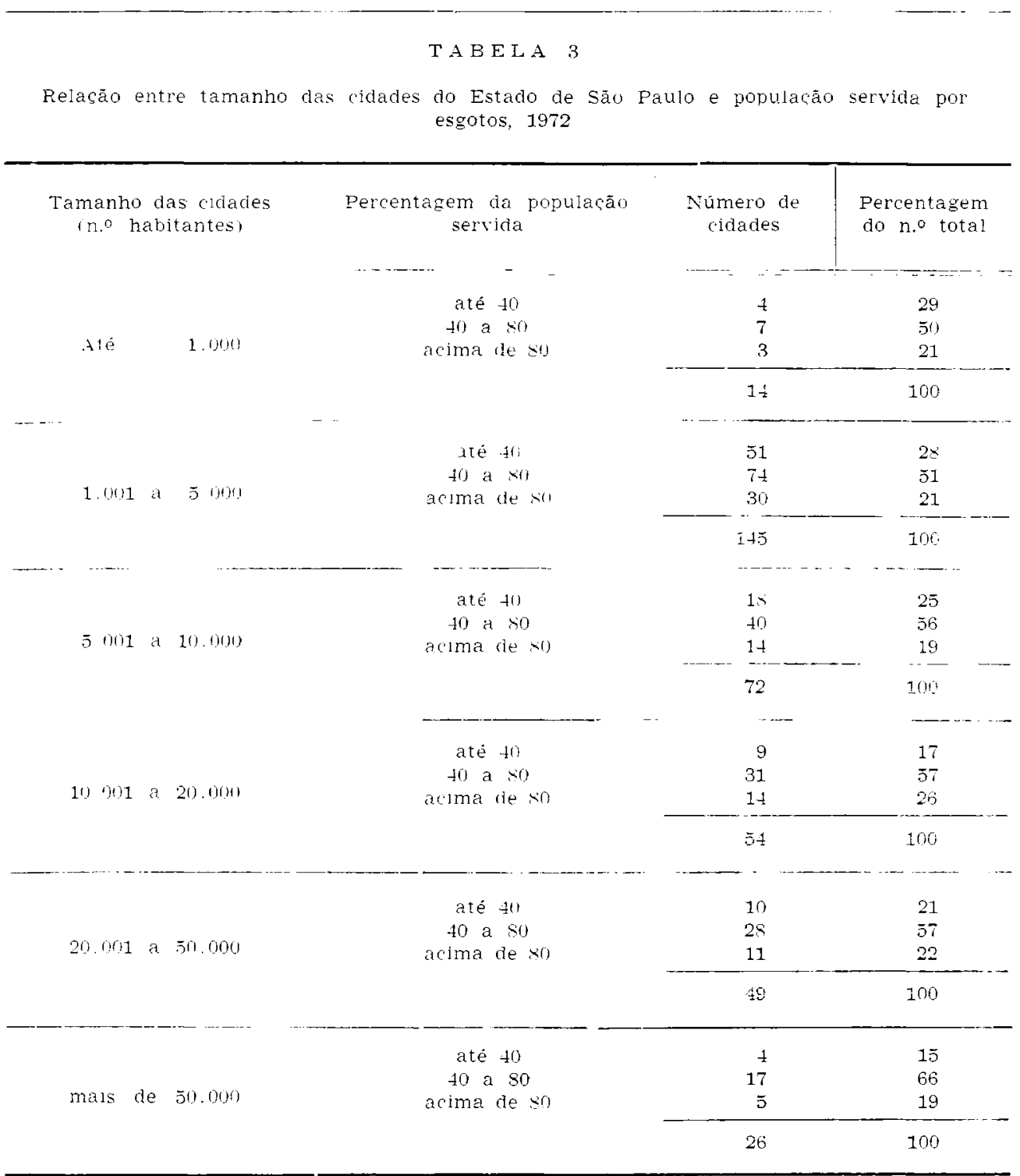

Fonte: Dados brutos, FESB

pulação urbana, cresce a dificuldade para que metas superiores de atendimento da coletividade sejam atingidas.

O fato já apresentado. de que nos grandes centros urbanos situa-se naturalmente a maioria dos recursos assistenciais mais sofisticados. permite evidentemente. um melhor atendimento de sua população. Contudo. a existência desse equipamento disponível faz com que elementos de outras áreas procurem os centros, agravando a sobrecarga assistencial que eles já enfrentavam e distorcendo os números e as estatísticas correspondentes. É o que nos mostra a Tahela 4. 
LIMA GONÇALVES, E. et al. - Problemas de assistência médico-sanitária no Estado de São Paulo, Brasil. Rev. Saúde públ., S. Paulo, 9:181-90, 1975.

TABELA 4

Total de óbitos registrados e óbitos de residentes fora dos municipios, segundo a procedência, 1969

\begin{tabular}{|c|c|c|c|c|c|c|c|}
\hline \multirow{3}{*}{ Cidade } & \multirow{3}{*}{$\begin{array}{l}\text { obitos } \\
\text { registra- } \\
\text { dos }\end{array}$} & \multicolumn{6}{|c|}{ obitos de fora do municipio } \\
\hline & & \multicolumn{2}{|c|}{ Total } & \multicolumn{2}{|c|}{ Mesma região } & \multirow{2}{*}{$\frac{\text { Outras }}{\text { N.o }}$} & \multirow{2}{*}{$\frac{\text { regiōes }}{\%}$} \\
\hline & & N.o & $\%$ & N.o & $\%$ & & \\
\hline São Paulo & 48.616 & 7.045 & 14,5 & 4.512 & 9,3 & 2.170 & 4,5 \\
\hline Santos & 4.238 & 697 & 16,4 & 647 & 15,2 & 45 & 1,1 \\
\hline S. José dos Campos & 1.341 & 134 & 10,0 & 63 & 4,7 & 60 & 4,5 \\
\hline Sorocaba & 1.779 & 280 & 15,7 & 255 & 14,3 & 22 & 1,2 \\
\hline Campinas & 3.125 & 534 & 17,1 & 412 & 13,2 & 84 & 2,7 \\
\hline Ribeinão Preto & 2.014 & 576 & 28,6 & 428 & 21,3 & 102 & 5,1 \\
\hline Bauru & 1.210 & 41 & 3,4 & 25 & 1,9 & 16 & 1,3 \\
\hline S. José do Rio Preto & 1.160 & 141 & 12,2 & 116 & 10,0 & 18 & 1,6 \\
\hline Araçatuba & 822 & 79 & 9,6 & 58 & 7,0 & 13 & 1,6 \\
\hline Presidente Prudente & 950 & 300 & 31,6 & 212 & 22,3 & 19 & 2,0 \\
\hline Marilia & 1.036 & 99 & 9,6 & 69 & 6,7 & 30 & 2,9 \\
\hline
\end{tabular}

* Inclui óbitos de municipios não especificados

Fonte: Guedes ${ }^{1}$

Outra linha de problemas médico-assistenciais é representada pelo atendimento da população rural. Aqui a evolução é representada por uma etapa inicial em que a atenção médica era oferecida, em caráter precário e em geral gratuito, por instituições hospitalares basicamente correspondentes às Santas Casas de Misericórdia. Em etapa posterior, procurou-se estender aos trabalhadores rurais a assistência médica previdenciária, por meio de "Estatuto do Trabalhador Rural" que, em 1963, criou o Fundo de Assistência e Previdência ao Trabalhador Rural - Funrural.

Esta iniciativa previu uma série de encargos bastante onerosos, tendo como fonte de receita a arrecadação correspondente a $1 \%$ do valor dos produtos agropecuários vendidos, o que rapidamente mostrou-se insuficiente.

Em 1971, o Programa de Assistência ao Trabalhadór Rural - Prorural veio alterar a situação; mais realista do que o programa anterior, modificou o esquema de custeio do plano, bem como as prestações a que os beneficiários tềm direito. O esquema básico de financiamento passou a corresponder à arrecadação de $2 \%$ sobre o valor comercial dos produtos rurais, ao lado de uma contribuição da indústria urbana, da ordem de $2,6 \%$, dos quais $2,4 \%$ são destinados ao Funrural.

Os serviços a serem prestados compreendem as áreas de saúde e de assistência social. Em relação àquelas, uma primeira diretriz básica do Prorural é prestá-los mediante convênios com entidades hospitalares ou ambulatoriais ou por meio de subsídios e eventuais doações de equipamentos. O regulamento é mesmo expresso em afirmar que, em nenhum caso, poderá o Funrural contratar ou manter pessoal para a realização de qualquer forma de prestação de serviços de saúde do trabalhador rural ou a seus dependentes.

Os serviços de saúde compreendem: prevenção de doenças, educação sanitária, assistência à infância e à maternidade, atendimento médico ou cirúrgico em ambulatório, hospital ou mesmo domicí- 
LIMA GONÇALVES, E. et 21. - Problemas de assistência médico-sanitária no Estado de São Paulo, Brasil. Rev. Saúde públ., S. Paulo, 9:181-90, 1975.

lio. exames complementares, assistência odontológica.

A segunda diretriz hásica corresponde à gratuidade que é assegurada, em princípio. aos assalariados; para os demais heneficiários do programa, especialmente para o produlor. abrangendo esta expressão as diversas figuras dos não assalariados que trahalham no campo, os serviços de saúde serão parcialme'nte custeados por estes.

Toda essa legrislação aparatosa paradoxalmente não melhorou a condição social do trabalhador rural. A razão fundamental é que os empregadores, temerosos dos encargos, passaram a rejeitar os empregados permanentes, dando preferência aos chamados "volantes" ou "boias frias"; em conseqüência, os trabalhadores rurais passaram a deslocar-se ainda mais intensamente para a cidade, agravando as condiçōes do proletariado urbano e estimulando a "favelização".

A avaliação global dos problemas médico-sanitários do Estado de São Paulo permite identificar $\tilde{j}$ grandes regiões, cujas situações diferem entre si.

A primeira dessas regiōes corresponde às áreas de São Joé do Rio Preto ${ }^{10}$, Araçatuba ${ }^{11}$ e Presidente Prudente ${ }^{12}$, cujas condições sócio-econômicas e de distrihuição de recursos de saúde podem ser assim resumidas: taxas de urbanização de 54,57 e $51 \%$ respectivamente, contra $80 \%$ do Estado como um todo; estrutura de produção predominantemente primária; saldo migratório líquido negativo; subregistro de óbitos na região rural; déficit de $30,1 \%$ no abastecimento de água e de $58,8 \%$ nas ligações à rede de esgotos; relação média de leitos hospitalares gerais de 2,5 e de leitos hospitalares especializados de 0,92 por mil habitantes; relação de componentes básicos de equipe de saúde (médico, dentistas, farmacêuticos, enfermeiras diplomadas) de 0,47 por mil habitantes; relação de servidores por leito hospitalar de 0,51 .

Os principais aspectos da situação médico-hospitalar desta região são: haixos coeficientes de mortalidade geral $\quad(6.7-6.3$ c 5,9 por mil hahitantes respectivamente, nas áreas de São José do Rio Preto. Araçatuba e Presidente Prudente contra 8,02 para o Estado); haixos coeficientes de mortalidade infantil (69.9-69.5 $e^{\circ}$ 74,4 por mil nascidos vivos respectivamente para as 3 áreas. contra 84.4 para lodo o Estado); apreciável incidência de gastrenterites e doenças infecciosas e parasitárias (febre tifóide. disenterias, luleerculose) entre as principais causas do obito; apreciável suscetibilidade da população a moléstias transmissíveis controláveis pela vacinação (difteria, tétano, sarampo).

A segunda região corresponde às áreas de Bauru ${ }^{9}$ e Marília ${ }^{13}$, onde as condições sócio-econômicas e de distribuição de recursos de saúde podem ser assim resumidas: taxas de urbanização de 68 e $56 \%$ respectivamente; saldo migratório líquido negativo; déficit de $21,5 \%$ no abastecimento de água e $43,1 \%$ nas ligações à rede de esgotos na área de $\mathrm{Ma}$ rília; relação média de leitos hospitalares gerais de 3,78 e de leitos especializados de 2,96 por mil habitantes; relação de componentes de equipe de saúde de 0,63 por mil habitantes; relação de servidores por leito hospitalar de 0,43 .

Os principais aspectos da situação médico-sanitária desta região são: coeficienlte de mortalidade geral de 8,1 por mil habitantes comparável à do Estado como um todo; coeficientes elevados de mortalidade infantil $(100,4$ e 95,3 por mil nascidos vivos); alta incidência de gastrenterites entre as principais causas de obito.

A terceira região corresponde às áreas de Sorocaba", do Litoral " e do Vale do Paraiba ${ }^{5}$, cujas condições sócio-econômicas e de distribuição de recursos de saúde podem ser assim resumidas: elevados índices de urbanização nos municípios capitais das diferentes áreas (Sorocaba -. 96,6\%: Santos - 99,4\% e São José dos Campos - 89,5\%); saldo migratório líquiido positivo notadamente na região do 
I.IMA GONCALVES, E. et al. - Problemas de assistência médico-sanitária no Estado de Sảo Paulo, Brasil. Rev. Saúde públ., S. Paulo, 9:181-90, 1975.

litoral; déficit de $34.7 \%$ no abastecimento de água e de $59.6 \%$ nas ligações à rede de' esgotos; relação média de leitos hospilalares gerais de 3,23 e de leitos especializados de 1,93 por mil habitantes; relação de componentes da equipe de saúde de 0,63 por mil hahitantes; relação de stervidores por leito hospitalar de 0,67 .

Os principais aspectos da situação médico-sanitária desta região: altos coeficientes de mortalidade geral (9,5 para Sorocaba, 8,5 para o Litoral e 9,1 para - Vale do Paraíba) e de mortalidade infantil (102,2 para Sorocaba, 78.4 para o Litoral e 80,0 para o Vale do Paraíba); alta incidência de gastrenterites e moléstias parasitárias entre as causas de mortalidade; aumento progressivo de acidentes de veículos a motor como causa de morte; apreciável incidência de tuberculose, hanseniase e esquistossomose.

A quarta região corresponde às áreas de Campinas $^{7}$ e Ribeirão Preto ${ }^{s}$, onde se observam as seguintes condições sócio-econômicas e de distribuição de recursos de saúde: taxas de urbanização de ordem de $70 \%$, com saldo migratório líquido positivo para Campinas e negativo, embora pouco expressivo, para Ribeirão Preto; déficit de $16,9 \%$ no abastecimento de água e de $34,2 \%$ nas ligaçōes à rede de 'sgotos; relação média de leitos hospitalares gerais de 3,68 e de leitos especializados de 2,96 por mil habitantes; relação de componentes da equipe de saúde de 0,79 por mil habitantes; relação de servidores por leito hospitalar 0,54. Saliente-se que esses índices permitem caracterizar esta regiáo como a mais bem servida de recursos de saúde do Estado.

Os principais aspectos da situação médico-sanitária desta região são: coeficientes de mortalidade geral de 7,8 e 7,4 e de mortalidade infantil de 72,2 e 71,4 respectivamente para Campinas e Ribeirão Preto.

A quinta região corresponde à Grande São Paulo $^{3}$, onde as condições sócio-econômicas e de distribuição de equipamen- tos e recursos de saúde são: taxa de urhanização de $96 \%$, com elevado saldo migratório positivo (este foi predominantemente do sexo feminino e da faixa etária de 10 a 29 anos no município de São Paulo e do sexo masculino e faixa etária de 0 a 9 anos para os demais municípios); déficit de $52,4 \%$ no abastecimento de água e de 71,3\% nas ligaçõos à rede de esgotos; relação de leitos hospitalares gerais de 3,00 e de leitos especializados de 2,81 por mil habitantes; relação de componentes da equipe de saúcle de 0,93 por mil habitantes; relação de servidores por leito hospitalar de 0,99 . Saliente-se que o número total de médicos nesta região, oferece uma relação de 1,2 por mil habitantes, índice considerado satisfatório; observa-se, contudo, um déficit de $36,5 \%$ desses profissionais nas unidades sanitárias, o que pode ter reflexos na situação médico-assistencial.

Os principais aspectos da situação sanitária são: coeficientes de mortalidade geral de 8,2 e de mortalidade infantil de 91,5 contra 8,0 e 84,4 para o Estado $\mathrm{cm}$ geral; significativa freqüência de moléstias infecto-contagiosas (tétano, difteria, poliomielite, sífilis, tuberculose), com surtos apreciáveis de doenças (disenterias, febre tifóide), que retratam as precárias condições de saneamento básico da área. Saliente-se, como elemento de alta preocupação, que os coeficientes de mortalidade infantil vêm aumentando permanentemente nos últimos 10 ou 15 anos.

$\mathrm{Na}$ Tabela 5 estão reunidos os diferentes coeficientes enumerados para as cinco regiōes do Estado analisadas.

Para melhor ilustrar as dificuldades com que se defrontam os que analisam os níveis de saúde de uma comunidade, vale a pena procurar correlacionar os dados da Tabela 5 com os indicadores de saúde apresentados no texto, os quais correspondem ao conjunto de dados disponíveis para todas as regiōes do Estado de São Paulo. Para tanto, foi composta a Tahela 6. 
LIMA GONCALVES, E. et al. - Problemas de assisténcia médico-sanitária no Estado de São Paulo, Brasil. Rev, Saúde públ., S. Paulo, 9:181-90, 1975.

TABELA 5

Indicadores sócio-económicos e de recursos de saúde correspondente a diferentes regióes do Estado de São Paulo, 1970

\begin{tabular}{|c|c|c|c|c|c|}
\hline Indicadores & $\mid \begin{array}{l}\text { S. J. R Preto } \\
\text { Aracatuba } \\
\text { P. Prudente }\end{array}$ & $\begin{array}{l}\text { Bauru } \\
\text { Marilia }\end{array}$ & $\begin{array}{c}\text { Sorocaba } \\
\text { Litoral } \\
\text { V. Paraiba }\end{array}$ & $\begin{array}{l}\text { Campinas } \\
\text { Rib. Preto }\end{array}$ & $\begin{array}{l}\text { Grande } \\
\text { S. Paulo }\end{array}$ \\
\hline $\begin{array}{l}\text { Taxa média de } \\
\text { urbanizacão }\end{array}$ & $\mathbf{5 4 , 0} \%$ & $62.0 \%$ & $71,0 \%$ & $70,0 \%$ & $96,0 \%$ \\
\hline $\begin{array}{l}\text { Déficit de abastecimento } \\
\text { de água * }\end{array}$ & $30,1^{\prime}$ & $21,1 \%$ & $34,7 \%$ & $16,9 \%$ & $52,4 \%$ \\
\hline $\begin{array}{l}\text { Déficit ligacāo rede de } \\
\text { eggotos }\end{array}$ & $58,8 \%$ & $43,1 \%$ & $59,6 \%$ & $34,2 \%$ & $71,3 \%$ \\
\hline $\begin{array}{l}\text { Relação leitos gerais } \\
\text { por } 1.000 \text { hab. }\end{array}$ & 2,48 & 3,78 & 3,23 & 3,68 & 3,00 \\
\hline $\begin{array}{l}\text { Relaça leitos especiall- } \\
\text { zados por } 1.000 \text { hab. }\end{array}$ & 0,92 & 2,96 & 1,93 & 2,96 & 2,81 \\
\hline $\begin{array}{l}\text { Relaç̃o componentes } \\
\text { equipe saúde por } \\
1.000 \text { hab. ** }\end{array}$ & 0,47 & 0,63 & 0,63 & 0,79 & 0,93 \\
\hline $\begin{array}{l}\text { Relação Servidores por } \\
\text { lelto hospitalar }\end{array}$ & 0,51 & 0,43 & 0,67 & 0,54 & 0,99 \\
\hline $\begin{array}{l}* \quad \text { fndices referentes a } \\
* \quad \text { Compreende total de } \\
\text { Fonte: Secretaria de }\end{array}$ & $\begin{array}{l}\text { ero de pré } \\
\text { dicos, dentis } \\
\text { conomia e }\end{array}$ & $\begin{array}{l}\text { da ár } \\
\text { farma } \\
\text { ejament }\end{array}$ & $\begin{array}{l}\text { ana } \\
\text { s, enf }\end{array}$ & & \\
\hline
\end{tabular}

T A B E L A 6

Coeficientes de mortalidade geral e infantil nas diferentes regióes do Estado de São Paulo

\begin{tabular}{l|c|c}
\hline Reglōes e sub-regiōes & $\begin{array}{c}\text { Mortalidade geral } \\
\text { (por 1.000 habitantes) }\end{array}$ & $\begin{array}{c}\text { Mortalidade infantil } \\
\text { (por 1.000 nascidos vivos) }\end{array}$ \\
\hline São José do Rio Preto & 6,7 & 69,9 \\
Aracatuba & 6,3 & 69,5 \\
Presidente Prudente & 5,9 & $\mathbf{7 4 , 4}$ \\
\hline Baurú & 8,1 & $\mathbf{1 0 0 , 4}$ \\
Marilia & & 95,3 \\
Sorocaba & $\mathbf{9 , 5}$ & $\mathbf{1 0 2 , 2}$ \\
Litoral & $\mathbf{8 , 5}$ & $\mathbf{7 8 , 4}$ \\
Vale do Paraíba & 9,1 & $\mathbf{8 0 , 0}$ \\
\hline Campinas & $\mathbf{7 , 8}$ & $\mathbf{7 2 , 2}$ \\
Ribeirão Preto & $\mathbf{7 , 4}$ & $\mathbf{7 1 , 4}$ \\
\hline Grande São Paulo & 8,2 & $\mathbf{9 1 , 5}$ \\
\hline Estado de São Paulo & $\mathbf{8 , 0}$ & 84,4 \\
\hline
\end{tabular}

Fonte: SEPLAN, 1972 
LIMA GONÇALVES, E. et al. - Problemas de assistência médico-sanitária no Estado de Sāu Paulo, Brasil. Rev. Saúde públ., S. Paulo, 9:181-90, 1975.

O exame da Tabela 6 demonstra que duas regiôes, das cinco examinadas, apresentam coeficientes superiores às do Estado de São Paulo como um todo: a de São José do Rio Preto, Araçatuba e Presidente Prudente e a de Campinas e Ribeirão Preto. Contudo, a análise da Tabela 5 revela uma acentuada discrepância entre as duas regióes referidas, quanto a uma série de aspectos relativos à existência e à distribuição de recursos: a primeira das regiōes descritas, correspondente ao oeste do Estado (São José do Rio Preto, Araçatuba e Presidente Prudente) encontra-se em situação inferior em relação à região de Campinas e Riheirão Preto. A razão da discrepância deve ser procurada em outras variáveis, bem como na própria obtenção dos dados \& na elaboração de registros.

É indiscutivel, entretanto, que a apresentação resumida dos recursos de saúde apresentados na Tabela 5 demonstra um apreciável esforço de dotar a comunidade de equipamentos necessários a uma assistência médico-sanitária adequada. Os resultados, contudo, apreciados por meio dos indicadores habituais ou da simples vivência de cada um no momento em que se coloca a necessidade de recorrer aos diferentes equipamentos de saúde, mostram-se ainda insatisfatórios. Ao vulto dos recursos investidos na implantação e no custeio de todo este equipamento não tem correspondido, na realidade, a eficácia desejável e necessária.

Diferentes mecanismos podem ser invocados para explicar os baixos resultados obtidos. De um lado, pesa sohre as agências de saúde em geral a sobrecarga de uma grave deficiência de tipo gerencial; em outras palavras, ainda não se conseguiu transpor para este tipo de atividade governamental a racionalização de atividades que pode e deve caracterizar a execução de suas tarefas.

$\mathrm{Na}$ área médico-assistencial, por exemplo, a crescente demanda de serviços tem criado dificuldades à adaptação da estrutura previdenciária a novas situaçōes, constituindo um dos fatores condicionantes de um atendimento que ainda deixa muito a desejar.

No que concerne à assistência médicohospitalar de urgência, é evidente a fragmentação e a falta de racionalidade dos serviços oferecidos à população. Quando ela é prestada pela municipalidade, como decorrência de preceito legal. que não discrimina a população-alvo, com frequêencia verifica-se a adoção de uma atitude pouco razoável que procura selecionar os doentes que a solicitam, ao distinguí-los entre aqueles que têm cobertura previdenciária e os que não a possuem. Esta situação decorre da falta de definição de atribuições que conduz a uma superposição inconveniente de atividades visando o mesmo objetivo.

Mas, existe outra série de razões que nos parece dominante; é a total falta de coordenação que se observa no desenvolvimento das atividades dos diferenies setores de saúde. Multiplicam-se as iniciativas paralelas. que desgastam os recurso: e escasseiam as atividades integradas. memo diante de situaçōes que afligem a comunidade. Este representa o principal problema médico-sanitário a ser enfrentado, porque sua solução deverá envolver a coordenação obrigatória das atividades desenvolvidas por diferentes estruturas vinculadas ao poder público federal. estadual ou municipal ou à iniciativa particular. 
LIMA GoNÇALVES, E. et al. - Problemas de assistência médico-sanitária no Estado de São Paulo, Brasil. Rev. Saúde públ., S. Paulo, 9:181-90, 1975.

Lima Gonçalves, E. et al. - [Problems of medical and sanitary attention in the State of S. Paulo, Brazil]. Rev. Saúde públ., S. Paulo, 9:181-90, 1975.

SUMMARY: The authors analyse the main medical and sanitary problems in the Brazilian State of $S$. Paulo and the possibility of defining, within the State, five zones, whose characteristics are outstandingly homogeneous: a first zone, including the regions of $S$. José do Rio Preto, Araçatuba and Presidente Prudente; a second zone, including Bauru and Marilia; a third zone, including the regions of Sorocaba, the coastal areas and the Paraiba Valley; a fourth zone including Campinas and Ribeirāo Preto and a fifth zone, represented by Greater S. Paulo. For each zone, the authors outline the basic health indices and some aspects of the available health facilities, concluding that it is difficult to establish a correlation between those two parameters. They also analyse the basic problems concerning the medical care of the S. Paulo State urban and rura? younlations.

UnITERMS: Health oure. Health level, indicators. S. Paulo, Brazil.

\section{REFERENCIAS BIBLIOGRAFICAS}

1. GUEDES, J. da S. - Contribuigio para - estudo da evolución lo nivel de saúde do Estado de São Paulo: análise das regioses administrativas, 1950 1970. São Paulo, 1972. [Tese de doutoramento - Faculdade de Saúde Pública da USP]

2. SAO PAULO. Secretaria de Economia e Planejamento. Diagnóstico do Etstado de Sño Paulo. São Paulo, 1973.

3. SÃO PAULO. Secretaria de Economia e Planejamento. Diagnóstico da $1 . "$ Regiño Administrativa. São Paulo. 1973.

4. Ibidem, 2.a Região Administrativa, São Paulo, 1972.

5. Ibidem, 3.a Regiāo Administrativa, São Paulo, 1972.

6. Ibidem, 4a Regiāo Administrativa. São Paulo, 1972.
7. Ibidem, 5.a Região Administrativa. Sãn Paulo, 1972.

8. Ibiđem, 6. Região Administrativa. São Paulo, 1972.

9. Ibidem, 7.a Região Administrativa. São Paulo, 1972.

10. Ibidem, 8. Região Administrativa. São Paulo, 1972.

11. Ibidem, 9.a Região Administrativa. São Paulo, 1972 .

12. Ibidem, 10.a Região Administrativa São Paulo, 1971 .

13. Ibidem, 11.a Região Administrativa. São Paulo, 1971.

Recebialo para publicacion em 12-08-1975 A provado para publicació em 0.4-0.4-1975 